\title{
Patient motion correction for dynamic cardiac PET: Current status and challenges
}

\author{
Yihuan Lu, a and Chi Liu ${ }^{\mathrm{a}}$ \\ a Department of Radiology and Biomedical Imaging, Yale University, New Haven, CT
}

Received Oct 29, 2018; accepted Oct 29, 2018

doi: $10.1007 / \mathrm{s} 12350-018-01513-\mathrm{x}$

\section{See related article, pp. 1982-1998}

For dynamic cardiac PET of quantifying myocardial blood flow (MBF), patient motion is a major factor that affects the ROI definition and absolute quantification accuracy. In a recent study, an ${ }^{82}$ rubidium $\left({ }^{82} \mathrm{Rb}\right)$-dynamic-tailored motion-correction framework has been proposed to address the voluntary body motion for all the dynamic frames, including both early and late phases. ${ }^{1}$ This approach brings us one step closer to the practical and full motion correction for dynamic cardiac PET studies. In this editorial, we discussed the current status and limitations of motion-correction methods for dynamic cardiac PET, including the recent publication at JNC, and also pointed out the remaining challenges for future developments.

PET myocardial perfusion imaging has been shown to improve the detection accuracy of coronary artery disease as compared to other non-invasive imaging modalities. ${ }^{2}$ Many investigators, in the past two decades, have established methods for absolute quantification of $\mathrm{MBF}$ and myocardial flow reserve (MFR) ${ }^{3}$ using dynamic PET, which is superior in diagnostic and prognostic value as compared to the conventional relative myocardial perfusion imaging. ${ }^{4}$

A prerequisite of accurate quantification in dynamic PET requires appropriate corrections ${ }^{5}$ to the original dynamic frame data. In additional to corrections for physical factors such as attenuation, scatter, randoms, and normalization, physiological factors such as patient motion also require correction. Patient motion in cardiac

Reprint requests: Chi Liu, Department of Radiology and Biomedical Imaging, Yale University, 789 Howard Ave., New Haven, CT 06519-1368; chi.liu@yale.edu

J Nucl Cardiol 2020;27:1999-2002.

1071-3581/\$34.00

Copyright (C) 2018 American Society of Nuclear Cardiology. imaging typically includes respiratory motion, cardiac motion, and voluntary body motion. Such motions can cause inaccurate tracer distribution estimation, as well as artifacts introduced by the mismatch between PET and CT-based attenuation map. ${ }^{6}$ Comparing to more periodic respiratory motion and cardiac motion, the timing for voluntary body motion is typically unpredictable, thus its impact on the MBF and MFR quantifications can be complicated. ${ }^{7}$

Methods for respiratory motion ${ }^{8}$ and cardiac motion management ${ }^{9}$ have been proposed in the past. The body motion correction has also been studied for several PET applications, especially for brain studies. ${ }^{10}$ However, the correction of body motion for ${ }^{82} \mathrm{Rb}$ cardiac dynamic PET imaging still remains highly challenging mainly due to the following two reasons. First, the rapid tracer kinetics of ${ }^{82} \mathrm{Rb}$ leads to substantial tracer distribution change in the dynamic images over time. Such spatial variations can lead to inaccurate motion estimation, where image registration is typically used, between dynamic frame images. The accuracy of image registration usually relies on the similarity between the two to-be-registered images. However, rapid tracer kinetics may result in one frame image very different from the other, therefore leading to inaccurate registration and subsequently inaccurate motion estimation. This might be the reason that currently available software in the clinic typically are only applicable to correct body motion in the later uptake phases ${ }^{11}$ but not early dynamic phases. Second, it remains challenging to detect body motion, where exact motion timing during the PET scan is hard to predict. The most commonly used frame-based registration approach can only correct motions between pre-defined dynamic frames, but cannot detect intra-frame motion, which is defined as the motion within a dynamic frame. For motion detection at a high temporal resolution, an external motion tracking system can be used. ${ }^{12}$ However, such tracking systems typically require extra setup time. Therefore, to facilitate clinical translation of body motion correction, data- 
driven motion detection methods without the need of external devices are preferred.

The work by Lee et al $^{1}$ published in this issue of JNC attempted to address some of the challenges described above. The authors established a body motioncorrection framework for dynamic ${ }^{82} \mathrm{Rb}$ cardiac PET. With extension from their previous studies, ${ }^{13,14}$ the current study addressed the needs of automated motion correction for the entire dynamic cardiac study, including both the late phase and the early blood pool phase with rapidly changing tracer kinetics. In this study, 225 patients underwent dynamic rest/stress ${ }^{82} \mathrm{Rb}$ PET imaging using a Siemens Biograph mCT PET/CT scanner, where pharmacological stress was performed with regadenoson. A 30-frame dynamic reconstruction, from 20 seconds since injection through 7 minutes, was performed using 3D-OSEM algorithm with pointspread-function and time-of-flight modeling. The investigators, without the ground truth, assumed good correlation in MBF/MFR with manual motion-correction results to be a measure of success. The strength of this premise relies on their previous studies, ${ }^{13}$ based on the same 225 patients cohort, that manual motion correction was considered as the gold standard when it was performed by a clinician for every dynamic frame. In Ref. 13, Lee et al found that translational body motion was most prevalent in the blood phase, resulting in the strongest impact on the right coronary artery region. Although only translational rigid motion was considered, the current work established an automated correction framework for cardiac dynamic PET especially including the blood pool phase, making it the only correction algorithm that applies to the entire dynamic sequence to the best of our knowledge.

In a dynamic PET study, after injection, ${ }^{82} \mathrm{Rb}$ in sequence enters right ventricle blood pool (RVBP), left ventricle blood pool (LVBP), and then myocardium tissue. Therefore, the tracer distribution in the early frames is very different from later frames. Thus, motion estimation between early and late frames can be recast as a multi-model registration problem, for which mutual information is typically used as the similarity metric. However, there has been no such application for the entire dynamic cardiac PET frames. The current study by Lee et $\mathrm{al},{ }^{1}$ instead, proposed to use a normalized gradient-field-based similarity metric, defined as the first order derivatives of a smoothed PET image, in the motion estimation process. With the assumption that "two image volumes were considered similar if the normalized gradient directions were aligned at a given position", , each dynamic image frame was rigidly aligned to match a fixed later phase summed frame, i.e., 120 to 400 seconds post-injection, to achieve motion correction. Incorporated with RVBP and LVBP regions generated by a blood pool-tissue isolation algorithm, this new similarity metric was tailored in this study to facilitate motion correction for the entire dynamic study.

In terms of motion-correction results, excellent linear agreement was found in MBF between the proposed automated motion-correction algorithm and manual motion correction. Effective reduction of the variability of MBF quantification was also achieved by using the automated algorithm. The proposed algorithm yielded consistent translational motion estimation with the manual motion-correction results for the tissue phase, albeit the motion was relatively small with less than $1 \mathrm{~mm}$ on average. The same agreement was not found in the blood phase, where the mean difference was found to be up to $5 \mathrm{~mm}$, mainly due to the fact that the clinician could not confidently estimate the motion for the RV blood phase in the manual approach. Nevertheless, the significance of the current study shall not only be judged by its correction effectiveness, but also by its innovation of the automated motion-correction framework, especially the motion estimation step using gradient-field-based similarity metric and its application to the entire dynamic study.

Of note, the overall $\mathrm{BM}$ magnitude reported in the current study was relatively small, even for the blood phase. Motion magnitude was found larger in the stress studies than that in the rest studies. ${ }^{1}$ In another study, the incidence and magnitude of motion reported in Ref. 7 were much larger than those reported in the current study. In Ref. 7, 24\% of the cases showed $0.5 \pm 0.1 \mathrm{~cm}$ body motion, $38 \%$ showed $1.0 \pm 0.3 \mathrm{~cm}$, and no difference was found between the rest and stress studies. In addition, in Ref. 7, substantial body motion maintained constant in frequency from 2 to 8 minutes while in the current study, minimal motion was found after 2 minutes post-injection. Both studies included over 200 patients thus the consideration of statistical significance is excellent. The motion magnitude and timing difference in the stress study could be explained by the use of a different vasodilator. ${ }^{15}$ In Ref. 15, patients stressed using 20 seconds-injected regadenoson was found to have a significantly lower amount of motion as compared to those with adenosine infused over several minutes. The current study ${ }^{1}$ used regadenoson while ${ }^{7}$ used dipyridamole. However, the reason for motion magnitude difference in the rest studies between the two publications is not clear. Note that all motion estimations in the above studies were based on human observation of pre-defined dynamic frames of reconstructions, which could be subjective and observerdependent. In addition, the motion within each of the dynamic frames, especially for later frames with longer durations, may be overlooked depending on the motion degree and timing. Therefore, if motion detection in 
Patient motion correction for dynamic cardiac PET

\section{Disclosure}

Yihuan Lu and Chi Liu have nothing to disclose related to this work.

\section{References}

1. Lee BC, Moody JB, Poitrasson-Rivière A, Melvin AC, Weinberg RL, Corbett JR, Murthy VL, Ficaro EP. Automated dynamic motion correction using normalized gradient fields for ${ }^{82}$ rubidium PET myocardial blood flow quantification. J Nucl Cardiol 2018. https://doi.org/10.1007/s12350-018-01471-4.

2. Prior JO, Allenbach G, Valenta I, Kosinski M, Burger C, Verdun FR, Bischof Delaloye A, Kaufmann PA. Quantification of myocardial blood flow with ${ }^{82} \mathrm{Rb}$ positron emission tomography: Clinical validation with 150 -water. Eur J Nucl Med Mol Imaging 2012;39:1037-47.

3. El Fakhri G, Sitek A, Guérin B, Kijewski MF, Di Carli MF, Moore SC. Quantitative dynamic cardiac ${ }^{82} \mathrm{Rb}$ PET using generalized factor and compartment analyses. J Nucl Med 2005;46:1264-71.

4. Ziadi MC, Williams K, Guo A, Renaud JM, Chow BJ, Klein R, Ruddy TD, Aung M, Garrard L, Beanlands RS. Does quantification of myocardial flow reserve using rubidium-82 positron emission tomography facilitate detection of multivessel coronary artery disease? J Nucl Cardiol 2012;19:670-80.

5. Kaufmann PA, Camici PG. Myocardial blood flow measurement by PET: Technical aspects and clinical applications. J Nucl Med 2005;46:75-88

6. Lu Y, Fontaine K, Mulnix T, Onofrey JA, Ren S, Panin VY, Jones J, Casey ME, Barnett R, Kench PL, Fulton R, Carson RE, Liu C. Respiratory motion compensation for PET/CT with motion information derived from matched attenuation corrected gated PET data. J Nucl Med 2018. https://doi.org/10.2967/jnumed.117. 203000.

7. Hunter CR, Klein R, Beanlands RS, deKemp RA. Patient motion effects on the quantification of regional myocardial blood flow with dynamic PET imaging. Med Phys 2016;43:1829.

8. Visvikis D, Lamare F, Bruyant P, Boussion N, Le Rest CC. Respiratory motion in positron emission tomography for oncology applications: Problems and solutions. Nucl Instrum Methods Phys Res A 2006;569:453-7.

9. Germino M, Carson RE. Cardiac-gated parametric images from $\mathrm{Rb}-82$ PET from dynamic frames and direct 4D reconstruction. Med Phys 2018;45:639-54.

10. Reilhac A, Merida I, Irace Z, Stephenson M, Weekes A, Chen C, Totman J, Townsend DW, Fayad H, Costes N. Development and validation of a rebinner with rigid motion correction for the Siemens PET-MR scanner: Application to a large cohort of $\left[{ }^{11} \mathrm{C}\right]$-PIB scans. J Nucl Med 2018. https://doi.org/10.2967/jnumed.117. 206375.

11. Pan X-B, Declerck J, Burckhardt D. Cardiac positron emission tomography: Overview of myocardial perfusion, myocardial blood flow and myocardial flow reserve imaging. Knoxville: Siemens; 2011.

12. Pretorius PH, Johnson KL, King MA. Evaluation of rigid-body motion compensation in cardiac perfusion SPECT employing polar-map quantification. IEEE Trans Nucl Sci 2016;63:1419-25.

13. Lee BC, Moody JB, Poitrasson-Riviere A, Melvin AC, Weinberg RL, Corbett JR, Ficaro EP, Murthy VL. Blood pool and tissue phase patient motion effects on ${ }^{(82)}$ rubidium PET myocardial blood flow quantification. J Nucl Cardiol 2018. https://doi.org/10. 1007/s12350-018-1256-1. 
14. Lee B, Moody J, Murthy V, Corbett J, Ficaro E. Image-based motion correction of the blood pool phase of dynamic PET data using blood pool isolation. J Nucl Med 2016;57:476.

15. Memmott MJ, Tonge CM, Saint KJ, Arumugam P. Impact of pharmacological stress agent on patient motion during rubidium82 myocardial perfusion PET/CT. J Nucl Cardiol 2018;25:128695.

16. Ren SL, Jin X, Chan C, Jian YQ, Mulnix T, Liu C, Carson RE. Data-driven event-by-event respiratory motion correction using TOF PET list-mode centroid of distribution. Phys Med Biol 2017;62:4741-55.

17. Thielemans K, Schleyer P, Marsden PK, Teuho J, Teras M, Bettinardi V, Menezes L, Manjeshwar RM, Stearns CW. Data-driven dual-gating for cardiac PET. In: 2014 IEEE nuclear science symposium and medical imaging conference (NSS/MIC), 2014.

18. Kolbitsch C, Ahlman MA, Davies-Venn C, Evers R, Hansen M, Peressutti D, Marsden P, Kellman P, Bluemke DA, Schaeffter T. Cardiac and respiratory motion correction for simultaneous cardiac PET/MR. J Nucl Med 2017;58:846-52.

19. Yu Y, Chan C, Ma T, Liu Y, Gallezot J-D, Naganawa M, Kelada OJ, Germino M, Sinusas AJ, Carson RE, Liu C. Event-by-event continuous respiratory motion correction for dynamic PET imaging. J Nucl Med 2016;57:1084-90.

20. Rezaei A, Michel C, Casey ME, Nuyts J. Simultaneous reconstruction of the activity image and registration of the CT image in TOF-PET. Phys Med Biol 2016;61:1852-74. 